\title{
Potentiometric Study of Dissociation Constants of Dihydroxybenzoic Acids at Reduced Ionic Strengths and Temperatures
}

\author{
Jörgen Rosenqvist, Caroline M. Jonsson \\ Department of Chemistry and Molecular Biology, University of Gothenburg, Gothenburg, Sweden \\ Email: jorgen.rosenqvist@chem.gu.se
}

How to cite this paper: Rosenqvist, J. and Jonsson, C.M. (2017) Potentiometric Study of Dissociation Constants of Dihydroxybenzoic Acids at Reduced Ionic Strengths and Temperatures. American Journal of Analytical Chemistry, 8, 142-150.

https://doi.org/10.4236/ajac.2017.82011

Received: December 8, 2016

Accepted: February 1, 2017

Published: February 4, 2017

Copyright $\odot 2017$ by authors and Scientific Research Publishing Inc. This work is licensed under the Creative Commons Attribution International License (CC BY 4.0).

http://creativecommons.org/licenses/by/4.0/

\begin{abstract}
The dissociation behavior of two dihydroxybenzoic acid isomers, 2,3-DHBA and 3,4-DHBA, at $281 \mathrm{~K}$ and $293 \mathrm{~K}$ was determined by potentiometric titrations in $0.01 \mathrm{M} \mathrm{NaCl}$ and $0.03 \mathrm{M} \mathrm{NaCl}$. Results showed that the dissociation enthalpy for the carboxylic group in DHBA is close to zero, resulting in dissociation constants that do not vary appreciably with temperature, whereas the dissociation constants for the first hydroxyl group vary significantly with temperature. Increasing ionic strength was found to result in increased values for the second dissociation constant, whereas the effect on the first dissociation constant was less clear.
\end{abstract}

\section{Keywords}

2,3-Dihydroxybenzoic Acid, 3,4-Dihydroxybenzoic Acid,

Dissociation Constant, pKa, Temperature, Ionic Strength, Surface Water

\section{Introduction}

Natural organic matter (NOM), such as humic acids, is ubiquitous in natural waters. Through complexation and adsorption processes, NOM determine the fate and behavior of particles and dissolved ions in water and it is therefore important to understand and be able to describe in detail the interactions between NOM and ions or particles. However, NOM consists of relatively large organic molecules, with varying structures depending on their origin, containing a multitude of functional groups and it is sometimes difficult to interpret results from experimental work involving NOM. Hence, smaller model molecules, often containing only carboxylic and/or phenolic groups, are frequently used to study these interactions. The present work is focused on dihydroxybenzoic acids (DHBA), small aromatic molecules that contain one carboxyl group and two phenolic 
groups each (Figure 1) and are thus well suited as model NOM molecules. Specifically, we studied 2,3-DHBA (a.k.a. Pyrocatechuic acid or Hypogallic acid), which is a catechol and salicylate derivative, and 3,4-DHBA (a.k.a. Protocatechuic acid), which is a catechol derivative. Due to the formation of intramolecular hydrogen bonds between the -COO-group and - $\mathrm{OH}$ group in the ortho position in 2,3-DHBA, the 2,3- and 3,4-DHBA molecules behave somewhat differently in aqueous solution, which is reflected in their speciation behavior [1].

Complexation of dihydroxybenzoic acids with metals can prevent hydrolysis [1] [2] and affect the mobility and bioavailability of the metals. Studies of DHBA complexation with various metal ions in solution can be found in the literature; e.g. beryllium [3] aluminium [1], yttrium [4], and copper [5]. Recently, the presence of 3,4-DHBA was also shown to affect the adsorption of the radionuclide $\mathrm{Eu}$ (III) to $\mathrm{Al}_{2} \mathrm{O}_{3}$ particles [6]. However, there is no uniform picture of how the two ligands behave under various conditions, not even with respect to the deprotonation of the ligands. Several sets of dissociation constants ( $\mathrm{pKa}$ values) have been reported (Table 1), although it is not always clear at what conditions these values were determined, since proper referencing is sometimes missing. For instance, the constants listed for 2,3-DHBA on the first row and for 3,4-DHBA on the last row of Table 1 can be found in many handbooks, both in print and online, but the origin of those values is somewhat unclear. In the case

(a)

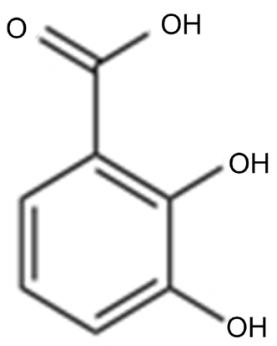

(b)

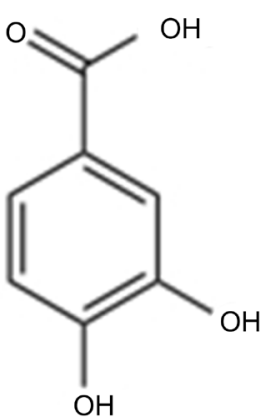

Figure 1. Structures of the fully protonated 2,3-DHBA (a) and 3,4-DHBA (b).

Table 1. Published dissociation constants (pKa values) for 2,3-DHBA and 3,4-DHBA.

\begin{tabular}{cccccccc}
\hline & $\mathrm{pKa}_{1}$ & $\mathrm{pKa}_{2}$ & $\mathrm{pKa}_{3}$ & Temp. (K) & Ionic medium & Method & Ref \\
\hline $2,3-$ & 2.41 & 10.30 & 13.48 & & & & N/A \\
DHBA & 2.74 & 9.91 & - & 298 & $0.1 \mathrm{M} \mathrm{NaClO}_{4}$ & Potentiometry & {$[7]$} \\
& - & 10.16 & - & 298 & $0.1 \mathrm{M} \mathrm{NaClO}_{4}$ & Spectroscopy & {$[7]$} \\
& 3.32 & 9.87 & $>14$ & 298 & $0.2 \mathrm{M} \mathrm{KCl}$ & Potentiometry & {$[1]$} \\
$3,4-$ & 4.22 & 8.65 & - & 298 & $0.6 \mathrm{M} \mathrm{NaCl}$ & Potentiometry & {$[8]$} \\
DHBA & 4.28 & 8.70 & 13.0 & 298 & $0.2 \mathrm{M} \mathrm{KCl}$ & Potentiometry & {$[1]$} \\
& 4.26 & 8.66 & - & 298 & $0.1 \mathrm{M} \mathrm{NaClO}$ & Potentiometry & {$[7]$} \\
& - & 9.06 & - & 298 & $0.1 \mathrm{M} \mathrm{NaClO}_{4}$ & Spectroscopy & {$[7]$} \\
& 4.48 & 8.83 & 12.6 & 298 & & & N/A \\
\hline
\end{tabular}


of 2,3-DHBA, $\mathrm{pKa}_{1}$ values ranging from 2.4 to 3.3 can be found, indicating a significant disagreement between different previous studies.

Furthermore, many of the reported dissociation constants were determined at relatively high ionic strength, and there is a substantial lack of information on the behavior of these two molecules at lower ionic strengths and at temperatures lower than $298 \mathrm{~K}$. Our main interest is in the interactions of organic molecules (e.g. DHBA) with $\mathrm{TiO}_{2}$ and $\mathrm{SiO}_{2}$ nanoparticles, as well as complexation of DHBA with metal ions (e.g. $\mathrm{Zn}^{2+}$ ) followed by adsorption onto nanoparticles in natural waters [9].

Thus, we need to know the speciation behavior of DHBA molecules at conditions relevant to natural waters and as a part of that decided to perform a detailed potentiometric study of the (de) protonation reactions of 2,3-DHBA and 3,4-DHBA in aqueous solution at two ionic strengths $(0.01 \mathrm{M}$ and $0.03 \mathrm{M} \mathrm{NaCl})$ and at two temperatures ( $293 \mathrm{~K}$ and $281 \mathrm{~K}$ ), covering an appreciable portion of the range of salinities and temperatures of typical Scandinavian stream and lake waters. The aim was to determine only the first two dissociation constants for each isomer, since the third dissociation step occurs at quite high $\mathrm{pH}$ (Table 1), where the dissociation constant cannot be determined with enough accuracy using common titration equipment, especially not at the low ionic strengths of this work.

\section{Materials and Methods}

All solutions were prepared from reagent grade chemicals and Milli-Q water (Millipore, resistivity $=18.2 \mathrm{M} \Omega \mathrm{cm}$ ). Concentrations of acids and bases were determined by titrations against known standard solutions. Potentiometric titrations of DHBA solutions were performed using a Metrohm 836 Titrando autotitrator, operated through Metrohm's Tiamo 2.3 data acquisition software package. The solution $\mathrm{pH}$ was measured with a Metrohm Aquatrode Plus combination electrode, filled with a saturated $\mathrm{KCl} / \mathrm{AgCl}$ solution, and calibrated before each titration at the temperature in question using standard $\mathrm{pH} 4$ and 7 buffers. A drift in measured potential of $\leq 0.1 \mathrm{mV} / \mathrm{min}$ was used as the equilibrium criterion, together with a fixed minimum equilibration time of $4 \mathrm{~min}(293 \mathrm{~K})$ or $5 \mathrm{~min}(281 \mathrm{~K})$. With these settings, total titration times varied from $7 \mathrm{~h}$ to $13 \mathrm{~h}$. Temperature (281 or $293 \mathrm{~K}$ ) was controlled to within $0.1 \mathrm{~K}$ using a double-jacketed titration vessel and a circulating water bath. An overhead propeller stirrer kept the solution homogeneous and the headspace was continuously purged with humid nitrogen gas to prevent $\mathrm{CO}_{2}$ contamination. A blank titration was performed before each DHBA titration, using identical solutions and the measured deviations from ideal behavior were subtracted from the raw data in the subsequent titration. At least three titrations with different DHBA concentrations (ranging from 0.0004 $\mathrm{M}$ to $0.0020 \mathrm{M}$ ) were performed at each set of conditions.

To simplify the evaluation of the titration results, the raw data was routinely recalculated into average number of protons adsorbed per DHBA molecule. The value of this variable, called $Z_{B}$, can be calculated for each titration point ac- 
cording to Equation (1):

$$
Z_{B}=\left(H-h+\left[\mathrm{OH}^{-}\right]\right) / B
$$

In Equation (1), $H$ is the total concentration of protons in the solution (calculated according to Equation (2) below), $h$ is the "free" concentration of protons in the solution (calculated according to Equation (3) below), $\left[\mathrm{OH}^{-}\right]$is the concentration of hydroxide ions in solution and $\mathrm{B}$ is the total concentration of DHBA in the solution (calculated according to Equation (4) below). All DHBA titrations were started by mixing a volume of DHBA solution $\left(V_{D}\right)$ with a volume of acid ( $\left.V_{a}\right)$ to achieve a suitable starting $\mathrm{pH}$ and the mixture was then titrated with a base. Therefore, the value of $\mathrm{H}$ at each titration point can be calculated as follows:

$$
H=\left(H_{D} \times V_{D}+H_{a} \times V_{a}+H_{t} \times V_{t}\right) /\left(V_{D}+V_{a}+V_{t}\right)
$$

where $H_{D}$ is the total concentration of protons in the DHBA stock solution, $H_{a}$ is the concentration of the acid (roughly $0.010 \mathrm{M}$ ), $H_{t}$ is the concentration of the $\mathrm{NaOH}$ titrant (roughly $0.010 \mathrm{M}$, i.e. $H_{t}=-0.010 \mathrm{M}$ ), and $V_{t}$ is the volume of titrant added at each titration point. The value of the $\mathrm{H}_{\mathrm{D}}$ parameter can be defined in various ways, depending on the exact nature of the study in question. In this work, we have set $H_{D}=3^{*} B_{D}$, where $B_{D}$ is the concentration of DHBA in the stock solution. With this definition of $H_{D}$, the value of the $Z_{B}$ parameter will range from (plus) three to zero, where a $Z_{B}$ value of three means that the DHBA molecule is in its fully protonated state and a $Z_{B}$ value of zero indicates that all three functional groups have been deprotonated.

The free concentration of protons, $h$, at each titration point was calculated from the measured $\mathrm{pH}$ value using Equation (3):

$$
h=10^{-\left(\mathrm{pH}+\log \gamma_{H^{+}}\right)}
$$

$\gamma_{H^{+}}$is the activity coefficient for protons and was calculated for each temperature and ionic strength using the Davies equation [10]. The concentration of hydroxyl ions was calculated analogously, using the measured $\mathrm{pH}$ value, the activity coefficient for $\mathrm{OH}^{-}$and the autoprotolysis constant of water at the conditions in question (determined according to [11]). The value of the parameter B was calculated using Equation (4):

$$
B=\left(B_{D} \times V_{D}\right) /\left(V_{D}+V_{a}+V_{t}\right)
$$

Since the added acid and titrant contains no DHBA, there is no need for a $B_{a}$ or $B_{t}$ term in Equation (4). The dissociation constants for each isomer of DHBA were determined by least-square optimization using the code Fiteql 2.0 [12].

\section{Results and Discussion}

Two typical sets of raw data from titrations of 2,3-DHBA are shown in Figure 2.

It is obvious that no dissociation constants can be evaluated from plots of this kind, as the only standout feature in this plot is the buffer minimum around $\mathrm{pH}$ 7 , whereas the (possible) occurrences of dissociation reactions around $\mathrm{pH} 3$ and 


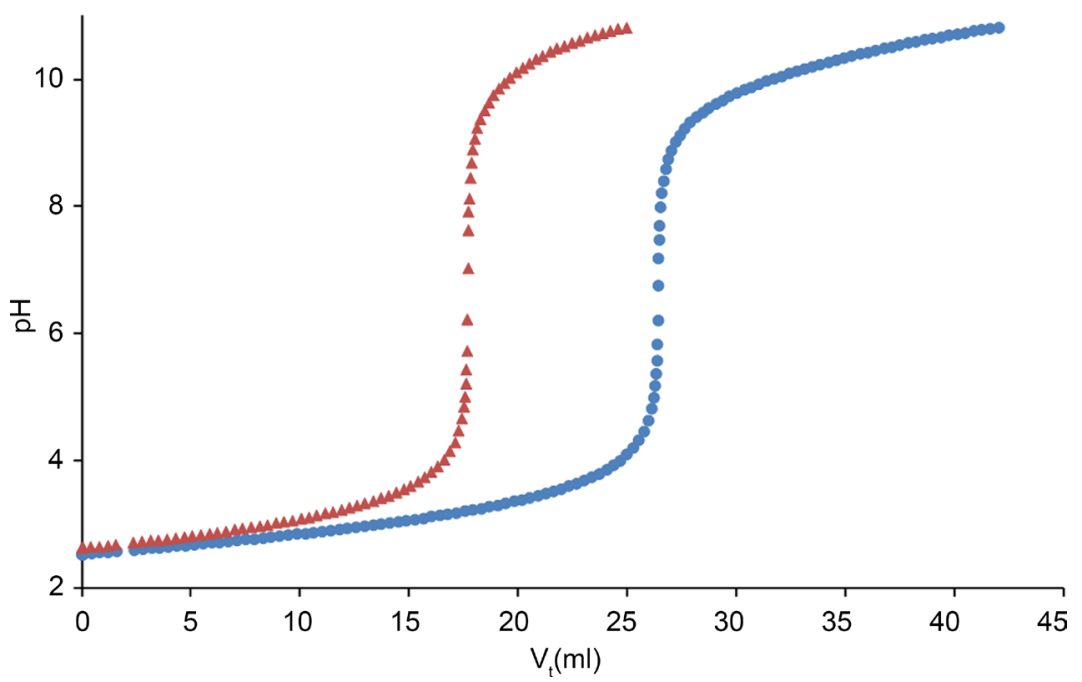

Figure 2. Raw data from titrations containing 0.0005 M 2,3-DHBA (red triangles) and $0.0019 \mathrm{M} 2,3$-DHBA (blue circles) in $0.03 \mathrm{M} \mathrm{NaCl}$ at $293 \mathrm{~K}$.

pH 10 are masked by the considerable buffer capacity of the aqueous solutions at those conditions. However, the dissociation behavior of the target (DHBA) molecules can be isolated from the buffering of the solvent by recalculating the raw data into the average number of protons adsorbed per DHBA molecule (i.e. $Z_{B}$ ) according to Equation (1). This reasonably straightforward mathematical treatment of the raw data allows for a much improved interpretation of the titration results, without the need for least-square fitting procedures etc. Typical $Z_{B}$ vs. $\mathrm{pH}$ curves for the two DHBA isomers are presented in Figure 3, which clearly illustrates the (de)protonation behavior of the DHBA molecules over the studied $\mathrm{pH}$ range, with the dissociation of the carboxylic group at acidic $\mathrm{pH}$ and the dissociation of the first hydroxyl group at basic $\mathrm{pH}$ clearly visible. The effect of ionic strength on the deprotonation behavior, especially for the second dissociation step, can also be detected in Figure 3. Converting the raw titration data into $Z_{B}$ curves thus simplifies the interpretation of the results considerably. In principle, the first two $\mathrm{pKa}$ values could be determined graphically from these $Z_{B}$ curves, as the pKas correspond to the $\mathrm{pH}$ values of $Z_{B}=2.5$ and $Z_{B}=1.5$, respectively. However, $Z_{B}$ curves can be somewhat sensitive to errors in the total concentration of the molecule in question (i.e. the B value in Equation (1)) and it is therefore advantageous to employ some type of fitting procedure, where both total concentrations and individual $\mathrm{pKa}$ values can be fitted simultaneously. In this work, the concentrations and pKas were fitted using Fiteql 2.0 and the values obtained using this code are listed in Table 2.

The larger uncertainties of (most) dissociation constants for 2,3-DHBA, compared to 3,4-DHBA (Table 2) are primarily due to the increase in uncertainty in $\mathrm{pH}$ measurements at low and high $\mathrm{pH}$, respectively. The results indicate that the first dissociation constant of 3,4-DHBA (for the carboxylic group) is quite insensitive to changes in conditions, the values are within uncertainty of each other, whereas the second dissociation constant (for the first hydroxyl group) shows considerable dependencies with both temperature and ionic 

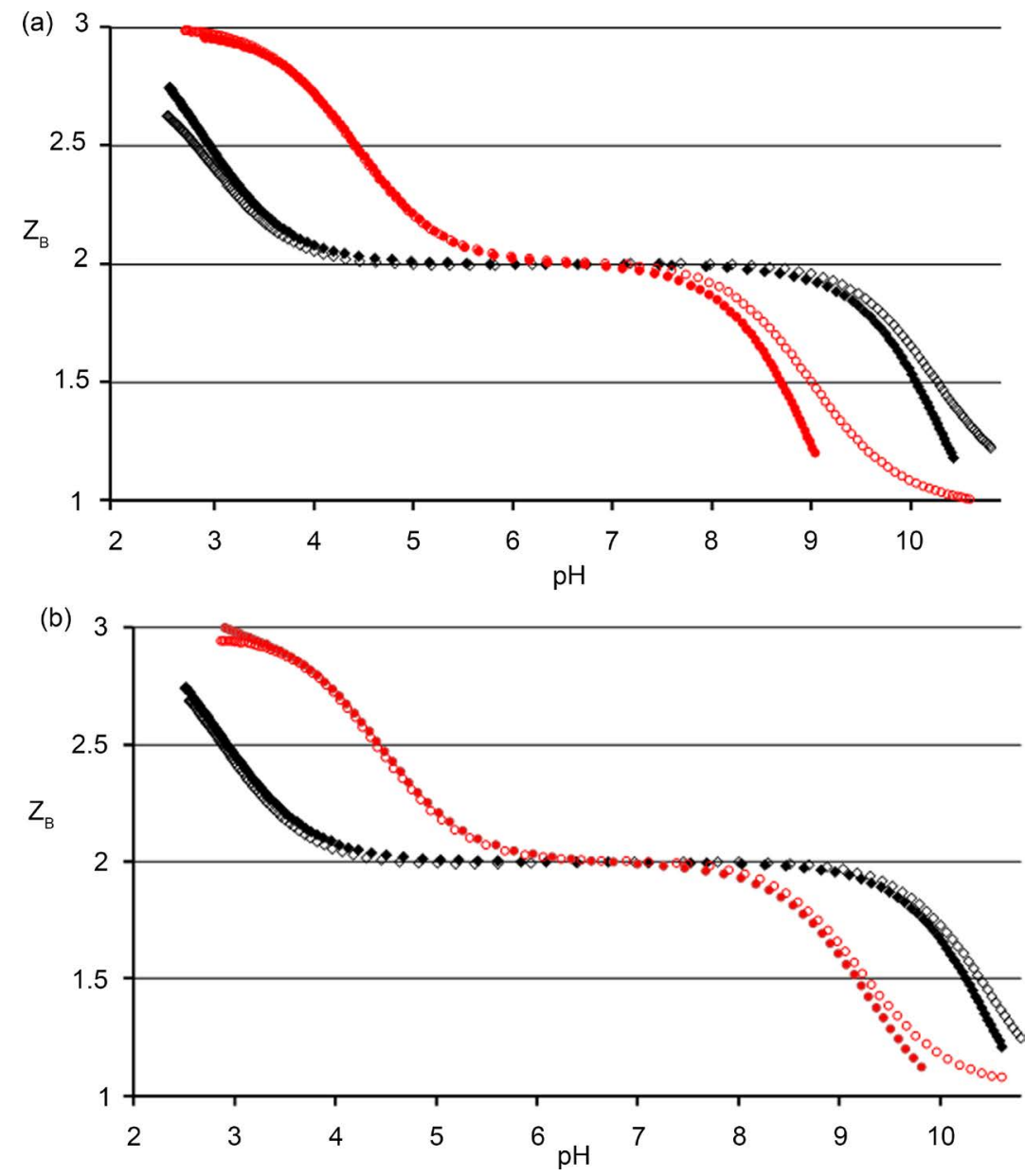

Figure 3. (a, b). $Z_{B}$-curves obtained at $293 \mathrm{~K}$ (a) and $281 \mathrm{~K}$ (b). Black diamonds show results for 2,3-DHBA while red circles show results for 3,4-DHBA. Filled symbols refer to titrations in $0.01 \mathrm{M} \mathrm{NaCl}$ and open symbols to titrations in $0.03 \mathrm{M} \mathrm{NaCl}$.

Table 2. Experimentally determined dissociation constants for 2,3-DHBA and 3,4-DHBA.

\begin{tabular}{ccccc}
\hline & {$[\mathrm{NaCl}](\mathrm{M})$} & Temp. $(\mathrm{K})$ & $\mathrm{pKa}_{1}( \pm 3 \sigma)$ & $\mathrm{pKa}_{2}( \pm 3 \sigma)$ \\
\hline 2,3 -DHBA & 0.01 & 293 & $2.93 \pm 0.05$ & $10.07 \pm 0.05$ \\
& 0.01 & 281 & $2.94 \pm 0.04$ & $10.26 \pm 0.02$ \\
& 0.03 & 293 & $2.81 \pm 0.05$ & $10.29 \pm 0.07$ \\
$3,4-\mathrm{DHBA}$ & 0.03 & 281 & $2.84 \pm 0.06$ & $10.44 \pm 0.03$ \\
& 0.01 & 293 & $4.42 \pm 0.02$ & $8.71 \pm 0.02$ \\
& 0.01 & 281 & $4.45 \pm 0.02$ & $9.16 \pm 0.02$ \\
& 0.03 & 293 & $4.40 \pm 0.03$ & $8.99 \pm 0.03$ \\
& 0.03 & 281 & $4.42 \pm 0.03$ & $9.26 \pm 0.03$ \\
\hline
\end{tabular}

strength. For 2,3-DHBA on the other hand, the first dissociation constant varies with ionic strength, but very little (if at all) with temperature.

The apparent independency of the pKa values for carboxylic groups with temperature shows that the dissociation enthalpy for the carboxylic group is 
close to zero. Similar behavior has previously been observed for some other organic molecules [13]. Hydroxyl groups on the other hand have larger dissociation enthalpies, resulting in clear increases in pKa values with decreasing temperatures [14]. The change in speciation with changing temperature is illustrated in Figure 4, where the distribution of different species over the investigated $\mathrm{pH}$ range is plotted. Since the first dissociation constant is insensitive to temperature change, the speciation of 3,4-DHBA will only vary with temperature if $\mathrm{pH}$ is above 6.5 , while for 2,3-DHBA temperature affects the speciation only above $\mathrm{pH}$ 8. $\mathrm{pH}$ values above 6.5 are unusual in stream and lake water, at least in Sweden [15]. Thus, seasonal variations in the speciation should be minimal.

The effect of ionic strength on the dissociation constant for the carboxylic

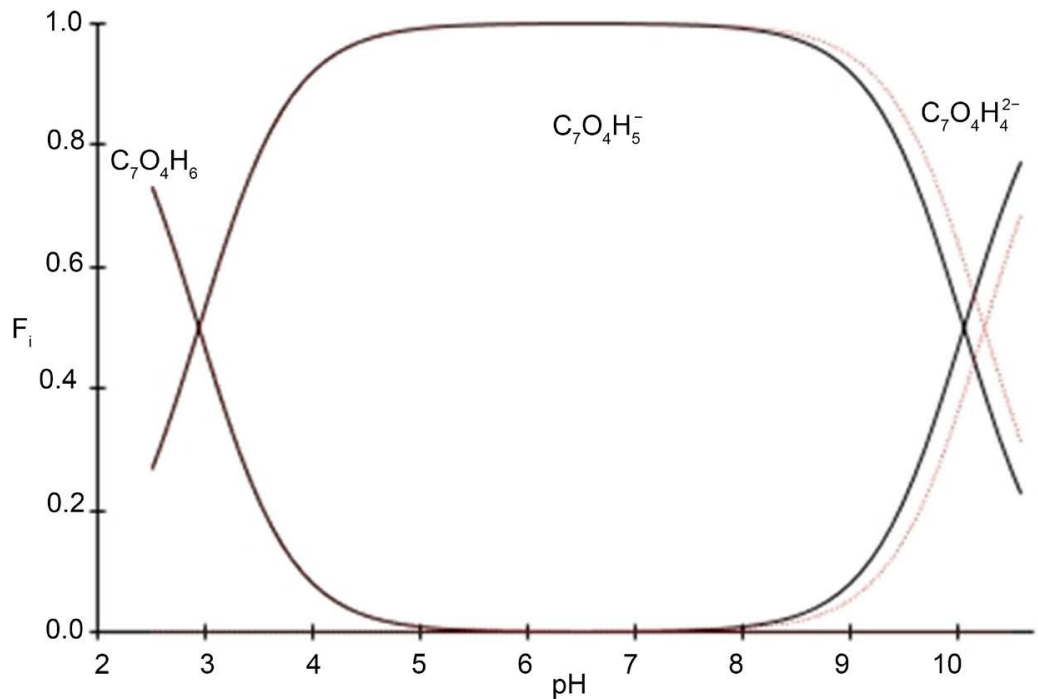

(a)

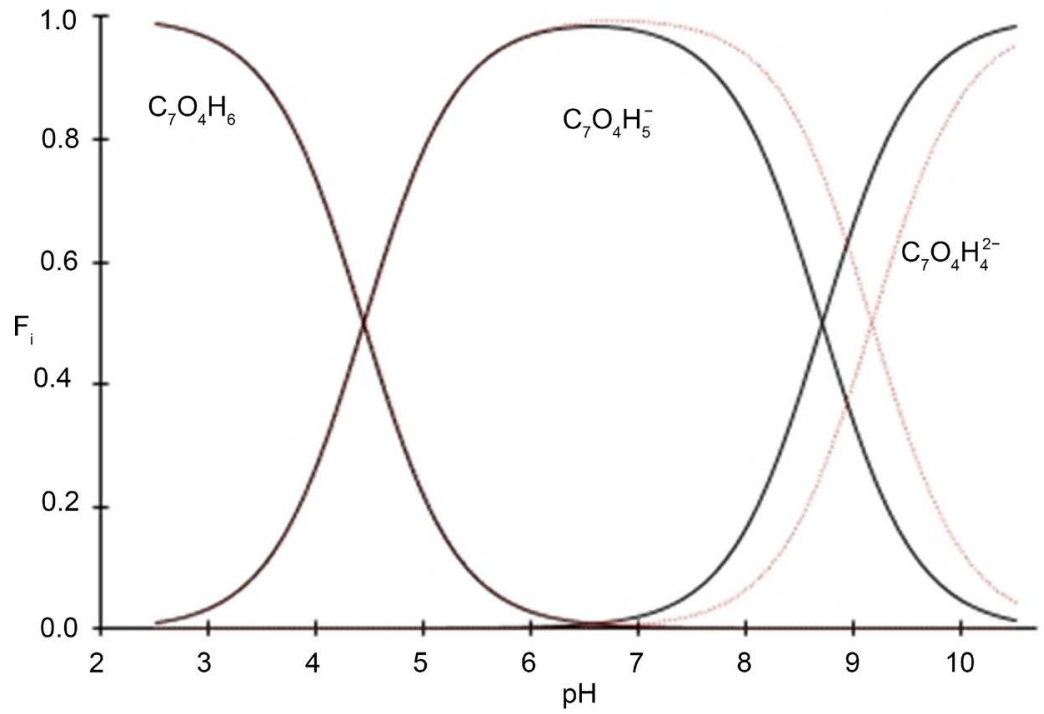

(b)

Figure 4. (a) and (b) Distribution diagram for 2,3-DHBA (a) and 3,4-DHBA (b) at $293 \mathrm{~K}$ (solid black lines) and $281 \mathrm{~K}$ (dotted red lines) in $0.010 \mathrm{M} \mathrm{NaCl}$ medium at atal DHBA concentration of $0.001 \mathrm{M} . F_{i}$ is defined as the ratio between the concentration of species $i$ and the total concentration of DHBA. 
groups is difficult to deduce from the present results, as the constant for 2,3DHBA seems to decrease with increasing ionic strength, whereas the shift is almost non-existent for 3,4-DHBA. Carboxylic groups usually exhibit decreasing dissociation constants with increasing ionic strength, at least at low to moderate ionic strengths [16], and it is not immediately clear why this trend is not more convincingly manifested in the case of 3,4-DHBA. Regarding the constant for the dissociation of the (first) hydroxyl group on the other hand, both isomers show the same behavior, with a considerable increase in the value of the constant with increasing ionic strength. This could somewhat complicate the use of DHBA as model molecules for humic acids and other forms of NOM, as it has been shown that at least for certain forms of humic acids, the dissociation constants for their hydroxyl groups decrease with increasing ionic strength [17].

\section{Conclusion}

The first dissociation constant for the two forms of DHBA showed negligible dependence on temperature, whereas the second dissociation constants increased considerably with decreasing temperatures. Changes in ionic strength on the other hand affect the two isomers in slightly different ways, with clear changes to the dissociation constants for 2,3-DHBA but near-negligible changes to the first dissociation constant of 3,4-DHBA.

\section{Acknowledgements}

We thank The Swedish Foundation for Strategic Environmental Research (Mistra) for financial support through the Mistra Environmental Nanosafety Program.

\section{References}

[1] Kiss, T., Atkári K., Jezowska-Bojczuk M. and Decock P. (1993) Complexes of Al(III) with Hydroxyaromatic Ligands. Journal of Coordination Chemistry, 29, 81-96. https://doi.org/10.1080/00958979308037128

[2] Martell, A.E., Motekaitis, R.J. and Smith, R.M. (1990) Aluminium Complexes of Hydroxyaliphatic and Hydroxyaromatic Ligands in Aqueous Systems-Some Problems and Solutions. Polyhedron, 9, 171-187. https://doi.org/10.1016/S0277-5387(00)80566-5

[3] Santoso, S.P., Angkawijaya, A.E., Soetaredjo, F.E., Ismadji, S. and Ju, Y.-H. (2015) Complex Equilibrium Study of some Hydroxyl Aromatic Ligands with Beryllium Ion. Journal of Molecular Liquids, 212, 524-531. https://doi.org/10.1016/j.molliq.2015.09.026

[4] Aydin, R. and Özer, U. (2004) Potentiometric and Spectroscopic Studies on Yttrium(III) Complexes of Dihydroxybenzoic Acids. Chemical and Pharmaceutical Bulletin, 52, 33-37. https://doi.org/10.1248/cpb.52.33

[5] Gerega, K., Kozłowski, H., Kiss, T., Micera, G., Strinna Erre, L. and Cariati, F. (1987) Cupric Complexes with 3,4-Dihydroxybenzoic Acid. Inorganica Chimica Acta, 138, 31-34. https://doi.org/10.1016/S0020-1693(00)81177-9

[6] Moreau, P., Colette-Maatouk, S., Gareil, P. and Reiller, P.E. (2016) Influence of Hydroxybenzoic Acids on the Adsorption of $\mathrm{Eu}(\mathrm{III})$ onto $\alpha, \gamma-\mathrm{Al}_{2} \mathrm{O}_{3}$ Particles in Mildly 
Acidic Conditions: A Macroscopic and Spectroscopic Study. Applied Geochemistry, 74, 13-23. https://doi.org/10.1016/j.apgeochem.2016.08.013

[7] Aydin, R., Özer, U. and Türkel, N. (1997) Potentiometric and Spectroscopic Determination of Acid Dissociation Constants of Some Phenols and Salicylic Acids. Turkish Journal of Chemistry, 21, 428-436.

[8] Fattahpour Sedeh, I., Öhman, L.-O. and Sjöberg, S. (1992) Equilibrium and Structural Studies of Silicon(IV) and Aluminium(III) in Aqueous Solution. 30. Aqueous Complexation between silicic Acid and Some ortho-Di- and Triphenolic Compounds. Acta Chemica Scandinavica, 46, 933-940.

https://doi.org/10.3891/acta.chem.scand.46-0933

[9] Rosenqvist, J. and Jonsson, C.M. (2015) Understanding the Structure of the Interface between $\mathrm{SiO}_{2}$ Nanoparticles and Organic Ligands. Goldschmidt Abstracts, 2015, 2688.

[10] Davies, C.W. (1962) Ion Association. Butterworths, London, 37-53.

[11] Sweeton, F.H., Mesmer, R.E. and Baes Jr., C.F. (1974) Acidity Measurements at Elevated Temperatures. VII. Dissociation of Water. Journal of Solution Chemistry, 3, 191-214. https://doi.org/10.1007/BF00645633

[12] Westall, J.C. (1982) FITEQL: A Computer Program for Determination of Chemical Equilibrium Constants from Experimental Data, Version 2.0. Report 82-02, Department of Chemistry, Oregon State University, Corvallis.

[13] Nagai, H., Kuwabara, K. and Carta, G. (2008) Temperature Dependence of the Dissociation Constants of Several Amino Acids. Journal of Chemical \& Engineering Data, 53, 619-627. https://doi.org/10.1021/je700067a

[14] Reijenga, J.C., Gagliardi, L.G. and Kenndler, E. (2007) Temperature Dependence of Acidity Constants, a Tool to Affect Separation Selectivity in Capillary Electrophoresis. Journal of Chromatography A, 1155, 142-145. https://doi.org/10.1016/j.chroma.2006.09.084

[15] Moldan, F., Cosby, B.J. and Wright, R.F. (2013) Modeling Past and Future Acidification of Swedish Lakes. AMBIO, 42, 577-586. https://doi.org/10.1007/s13280-012-0360-8

[16] Cohn, E.J., Heyroth, F.F. and Menkin, M.F. (1928) The Dissociation Constant of Acetic Acid and the Activity Coefficients of the Ions in Certain Acetate Solutions. Journal of the American Chemical Society, 50, 696-714. https://doi.org/10.1021/ja01390a012

[17] Badr, M.H., El-Halafawi, M.H. and Abd El-al Zeid, E.R. (2012) Comparison between the Effect of Ionic Strength on Acidity and Dissociation Constants of Humic Acids Extracted from Sewage Sludge and Nile Water Hyacinth Composts. Global Journal of Environmental Research, 6, 36-43. 
Submit or recommend next manuscript to SCIRP and we will provide best service for you:

Accepting pre-submission inquiries through Email, Facebook, LinkedIn, Twitter, etc. A wide selection of journals (inclusive of 9 subjects, more than 200 journals)

Providing 24-hour high-quality service

User-friendly online submission system

Fair and swift peer-review system

Efficient typesetting and proofreading procedure

Display of the result of downloads and visits, as well as the number of cited articles Maximum dissemination of your research work

Submit your manuscript at: http://papersubmission.scirp.org/

Or contact ajac@scirp.org 